\title{
Constraining Baryon-Dark-Matter Scattering with the Cosmic Dawn 21-cm Signal
}

\author{
Anastasia Fialkov \\ Harvard-Smithsonian Center for Astrophysics, 60 Garden Street, Cambridge, Massachusetts 02138, USA \\ Rennan Barkana and Aviad Cohen \\ Raymond and Beverly Sackler School of Physics and Astronomy, Tel Aviv University, Tel Aviv 69978, Israel
}

(Received 28 February 2018; revised manuscript received 25 April 2018; published 2 July 2018)

\begin{abstract}
The recent detection of an anomalously strong $21-\mathrm{cm}$ signal of neutral hydrogen from cosmic dawn by the EDGES low-band radio experiment can be explained if cold dark matter particles scattered off the baryons draining excess energy from the gas. In this Letter we explore the expanded range of the $21-\mathrm{cm}$ signal that is opened up by this interaction, varying the astrophysical parameters as well as the properties of dark matter particles in the widest possible range. We identify models consistent with current data by comparing to both the detection in the low-band region and the upper limits from the EDGES high-band antenna. We find that consistent models predict a $21-\mathrm{cm}$ fluctuation during cosmic dawn that is between 3 and 30 times larger than the largest previously expected without dark matter scattering. The expected power spectrum exhibits strong baryon acoustic oscillations imprinted by the velocity-dependent cross section. The latter signature is a conclusive evidence of the velocity-dependent scattering and could be used by interferometers to verify the dark matter explanation of the EDGES detection.
\end{abstract}

DOI: 10.1103/PhysRevLett.121.011101

Introduction.-The first few hundred million years after the big bang are the least explored period in the history of the Universe. This epoch is marked by some of the most interesting events in cosmic history such as the formation of the very first stars and black holes. However, what makes this epoch even more attractive for observers and theorists alike is that dark matter (DM) might have manifest itself differently than today in the unmatched physical conditions of the early Universe. Even though conventional DM models assume only gravitational interactions with ordinary baryonic matter, other forms of couplings, such as collisions between DM and gas, are not ruled out and could be important at early times when the density of matter was much higher than today. Such interactions could modify the thermal and ionization histories, leaving fingerprints in the 21-cm signal of atomic hydrogen [1-3].

In the standard cosmology, Compton scattering couples the baryon temperature, $T_{\text {gas }}$, to the temperature of the cosmic microwave background (CMB) at redshifts above $z_{\mathrm{dec}} \sim 200$. At lower redshifts, $T_{\text {gas }}$ is expected to cool adiabatically due to the expansion of the Universe until the moment when the first $\mathrm{x}$-ray sources turn on, injecting energy into the gas. Because DM is expected to decouple earlier and be much colder than the gas, collisions between baryons and DM particles could drain excess energy from the gas leading to its overcooling [1], while the relative velocity between DM and the gas could in some cases result in overall overheating of the baryons [2].

The $21-\mathrm{cm}$ line of neutral atomic hydrogen with a restframe frequency of $1.42 \mathrm{GHz}$ is one of the most promising probes of this epoch. This signal is sensitive to the thermal and ionization states of the baryons and, thus, can be used to measure the energy balance of the early Universe. The brightness temperature of the $21-\mathrm{cm}$ line, $T_{21}$, is coupled to the kinetic temperature of the baryons by two processes: During the cosmic dark ages $(35 \lesssim z \lesssim 1100$, the epoch preceding the formation of the very first stars) the gas is dense enough for interatomic collisions to drive the effective temperature of the $21-\mathrm{cm}$ transition to the temperature of the gas, a process that becomes less efficient as the Universe expands. During the subsequent epoch of cosmic dawn $(15 \lesssim z \lesssim 35)$ when the first stars are formed, the main driver is the Ly- $\alpha$ radiation produced by stars which couples the two temperatures via the Wouthuysen-Field process $[4,5]$. Because gas is colder than the background radiation at $z<z_{\mathrm{dec}}$ and before the first $\mathrm{x}$-ray sources turn on, the 21-cm signal from the dark ages and cosmic dawn is seen in absorption against the CMB. Features of the highredshift 21-cm signal depend on the underlying astrophysics [6], but also on the properties of DM particles, if the latter affect either the thermal or the ionization state of the gas [1-3]. Therefore, the $21-\mathrm{cm}$ signal can be used as a unique probe of the dark sector.

Although exploration of the high-redshift domain is one of the most active areas of research in astrophysics, properties of the early Universe are still poorly constrained. The uncertainty in astrophysical parameters and the limited understanding of the dark matter physics propagate into the $21-\mathrm{cm}$ modeling and result in a large variety of allowed signals. The dependence of the expected $21-\mathrm{cm}$ signal on 
astrophysical parameters has been extensively explored [6-8]. It is the goal of this Letter to explore the $21-\mathrm{cm}$ signal over the parameter space of both astro- and dark matter physics.

Observation and theory. - The first detection of the 21-cm signal from cosmic dawn was recently reported by the lowband antenna of the Experiment to Detect the Global EoR Signature (EDGES) [9] observing in the $50-100 \mathrm{MHz}$ frequency band and probing the $21-\mathrm{cm}$ signal from the redshift range of $z \approx 13.2-27.4$. After removal of the foregrounds and the instrumental noise, the excess signal was found in the data. The extracted cosmological signal, centered at $\nu=78 \pm 2 \mathrm{MHz}$ (which corresponds to $z=17.2$ ), features a broad absorption trough of $T_{21}=-500_{-500}^{+200} \mathrm{mK}$, where the error corresponds to $99 \%$ confidence including both thermal and systematic noise. In the standard cosmological scenario, the strongest possible absorption expected at $z \sim 17$ is $-209 \mathrm{mK}$, which corresponds to a gas temperature of $T_{\text {gas }} \sim 7 \mathrm{~K}$. The observed $T_{21}<300 \mathrm{mK}$ requires the gas to be much colder, $T_{\text {gas }}<5.1 \mathrm{~K}$, which is hard to explain by astrophysics alone [3]. The shape of the observed signal is also anomalous, with the best-fit profile featuring a flat bottom. So far, no plausible mechanism has been put forward to explain this feature. However, the detailed shape of the fit depends on the experimental setup (see Fig. 2 of Ref. [9]); thus, the significance of the flattening is unclear given the level of systematic error. In this Letter we therefore focus on the amplitude of the signal, leaving the shape of the profile for future work.

Feng and Holder [10] suggest that an excess radio background, such as seen by ARCADE 2 (Absolute Radiometer for Cosmology, Astrophysics and Diffuse Emission) [11], could produce anomalously strong absorption in the 21-cm signal at $z \sim 20$. However, the ARCADE 2 excess alone does not require astrophysical explanation and can be explained by carefully modeling the Galactic contribution [12].

To explain the anomalously strong absorption seen by EDGES low-band antenna, Barkana [3] invoked elastic velocity-dependent scattering between baryons and dark matter (baryon-DM scattering). The absorption trough as deep as detected by EDGES is obtained if baryons scatter off DM particles with masses in the range $m_{\chi}<4.3 \mathrm{GeV}$. The scattering cross section is assumed to be velocity dependent, $\sigma(v)=\sigma_{1}\left(v / 1 \mathrm{~km} \mathrm{~s}^{-1}\right)^{-4}$, where $\sigma_{1}>3.4 \times 10^{-21} \mathrm{~cm}^{2}$ and $v$ is the relative velocity between the baryon and the DM particle. Astrophysics also plays an important role, as the deep absorption is produced only in the presence of the stellar Ly- $\alpha$ background; the central frequency and the depth of the trough are determined, in addition to baryon-DM scattering, by the timing and intensity of both the Ly- $\alpha$ and the $\mathrm{x}$-ray radiative backgrounds.

A major role is played by the remnant baryon-DM relative velocity $v_{\text {b-DM }}$ from the early universe $[2,3]$. As a result of the velocity-dependent scattering, $T_{21}$ is expected to be modulated by the velocity field: regions where the velocity is low cool stronger and exhibit a stronger absorption signal; while regions where the velocity is high cool less. Examples of this dependence are shown in the top panels of Fig. 1 and the effect is discussed further in the text.

Cosmic dawn signal.-We model the $21-\mathrm{cm}$ signal as in Ref. [3] over the large parameter space of possible astrophysical and DM properties. To describe the astrophysics we use seven free parameters (see Ref. [8] for more details). Star formation is parameterized by two numbers: per-halo efficiency, $0.05 \% \leq f_{*} \leq 50 \%$, and minimum circular velocity (or, equivalently, the minimal cooling mass) of star forming halos, $4.2 \leq V_{c} \leq 76.5 \mathrm{~km} \mathrm{~s}^{-1}$. We use three parameters to describe the $\mathrm{x}$-ray population. These include the slope and the low-energy cutoff $\left(-1.5 \leq \alpha \leq-1,0.1 \leq \nu_{\min } \leq 3 \mathrm{keV}\right)$ of the spectral energy distribution, which is assumed to have a power law shape. The total x-ray luminosity is assumed to scale as the star formation rate and is parameterized by $f_{X}$ with $10^{-4} \leq f_{X} \leq 1000 ; f_{X}=1$ corresponds to the luminosity of low-redshift x-ray counterparts, with the redshift evolution of metallicity taken into account $[13,14]$. The ionizing efficiency of sources (calibrated to produce the total CMB optical depth consistent with the Planck data [15]) and the mean free path of ionizing photons $\left(20 \leq R_{\mathrm{mfp}} \leq 40\right.$ comoving Mpc) are the other two free parameters. We fix the cosmological parameters to the values measured by the Planck satellite [16]. For the study presented here we use a compilation of 6389 different astrophysical models populating the entire parameter space. For each astrophysical scenario we ran 51 models to include the DM physics. The baryon-DM scattering adds two parameters: the mass of DM particles, $0.0032<m_{\chi}<100 \mathrm{GeV}$, and the cross section $10^{-30}<\sigma_{1}<3.16 \times 10^{-18} \mathrm{~cm}^{2}$. The range of each of the parameters is consistent with existing observational limits [3].

In the absence of scattering, the $21-\mathrm{cm}$ signal is calculated using a state-of-the-art seminumerical code $[6,14]$. Given a set of astrophysical parameters, the simulation generates histories of the $21-\mathrm{cm}$ signal in comoving volumes of $384^{3} \mathrm{Mpc}^{3}$ resolved down to 3 comoving Mpc. On smaller scales, subgrid models are employed. Processes such as the growth of structure, star formation, heating, and ionization are incorporated. In the calculation, large scale structure is tracked from $z \sim 60$, just after the first stars are expected to form in the observable Universe, down to $z \sim 6$ when neutral gas is completely reionized. The reionization history is calibrated to observations [15]. Stars and stellar remnants produce inhomogeneous $\mathrm{x}$-ray, Ly- $\alpha$, LymanWerner, and ultraviolet radiative backgrounds. The impact of radiation on the environment is calculated, including heating of the intergalactic medium by $\mathrm{x}$ rays.

The baryon-DM scattering changes the energy budget of the gas. To calculate the complete $21-\mathrm{cm}$ signal we follow the approach taken by Barkana [3], which builds on the equations outlined by Muñoz et al. [2]. We calculate the gas 

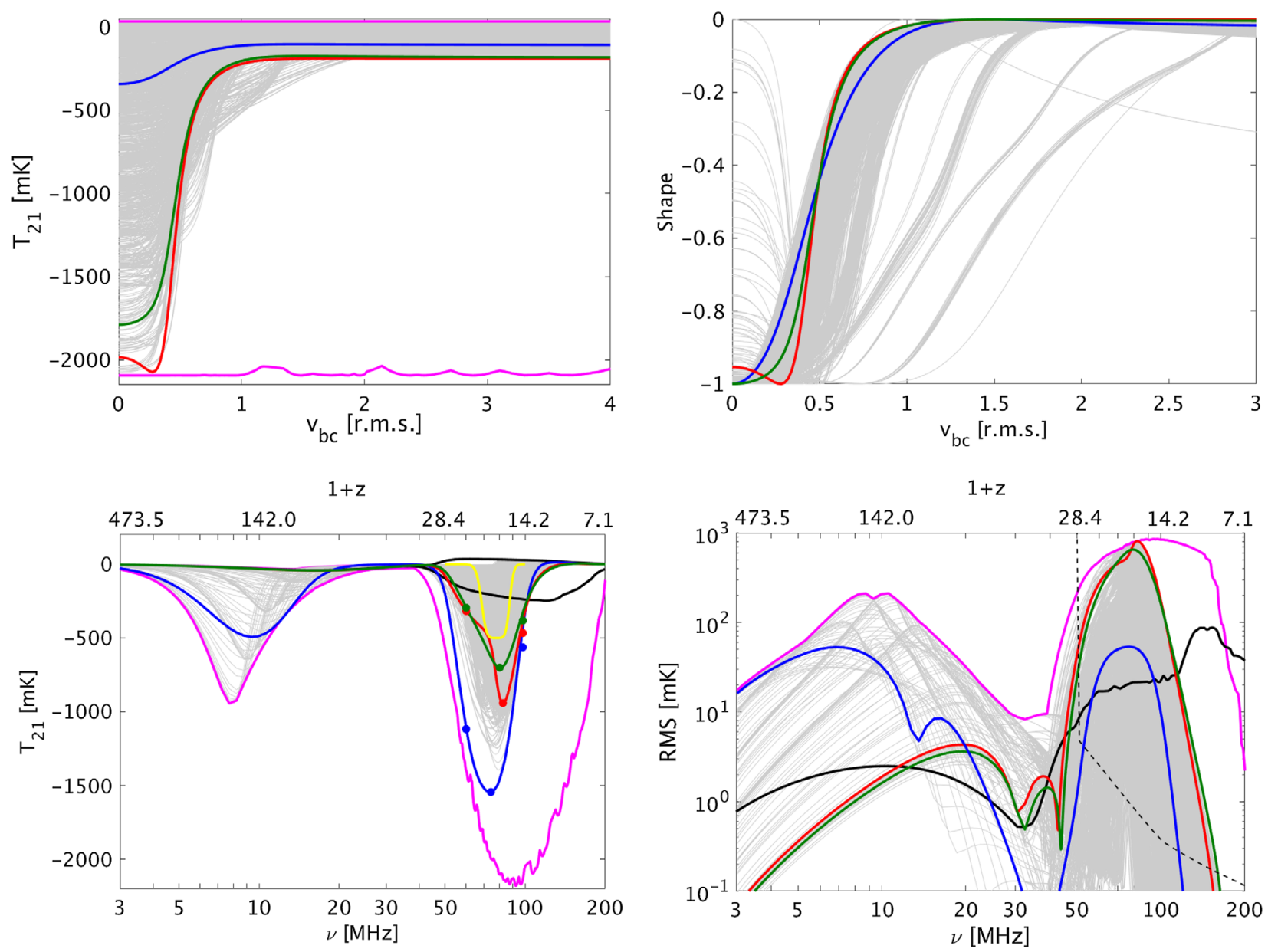

FIG. 1. Top: The function $T_{21}\left(v_{\mathrm{b}-\mathrm{DM}}\right)$ at $z=17$ in units of $\mathrm{mK}$ (left) and its shape (right) versus the local relative baryon-DM velocity $v_{\mathrm{b}-\mathrm{DM}}$ in units of the rms velocity. To show the shape we rescaled each $T_{21}\left(v_{\mathrm{b}-\mathrm{DM}}\right)$ along the $y$ axis to range from -1 to 0 . Bottom: Global (i.e., $v_{\mathrm{b}-\mathrm{DM}}$-averaged) 21 -cm signal (left) and rms of its fluctuations (right), as a function of frequency (bottom $x$ axis) or redshift (top $x$ axis). We show the envelope of all the possible signals without baryon-DM scattering (black, 6389 models) and with the scattering included (magenta, 325839 models). Also shown are all the models consistent [as defined in Eqs. (1)-(3)] with EDGES low-band and high-band data (gray lines, 2448 models in total). Out of the latter compilation we highlight three models: the model with the deepest absorption trough (blue) which is characterized by (see text) $V_{c}=16.5 \mathrm{~km} \mathrm{~s}^{-1}, f_{*}=0.5 \%, f_{X}=10, \alpha=-1, \nu_{\text {min }}=1 \mathrm{keV}$, $\tau=0.0703, R_{\mathrm{mfp}}=20 \mathrm{Mpc}, m_{\chi}=0.0032 \mathrm{GeV}$ and $\sigma_{1}=316 \times 10^{-20} \mathrm{~cm}^{2}$; the lowest redshift of heating transition (green) with $V_{c}=16.5 \mathrm{~km} \mathrm{~s}^{-1}, f_{*}=0.3 \%, f_{X}=0.0721, \alpha=-1, \nu_{\min }=1 \mathrm{keV}, \tau=0.0702, R_{\mathrm{mfp}}=30 \mathrm{Mpc}, m_{\chi}=0.1 \mathrm{GeV}$, and $\sigma_{1}=$ $1 \times 10^{-20} \mathrm{~cm}^{2}$; and the highest $\mathrm{rms}$ (red) with $V_{c}=16.5 \mathrm{~km} \mathrm{~s}^{-1}, f_{*}=0.5 \%, f_{X}=0.01, \alpha=-1, \nu_{\min }=0.1 \mathrm{keV}, \tau=0.0775$, $R_{\mathrm{mfp}}=20 \mathrm{Mpc}, m_{\chi}=0.0032 \mathrm{GeV}$, and $\sigma_{1}=1 \times 10^{-20} \mathrm{~cm}^{2}$. The min and max points from Eqs. (2) and (3) are shown for these three curves. Also shown are the best-fit signal to the EDGES low-band data [9] (yellow, bottom left); and the rms of the thermal noise for the SKA1 at $k=0.1 \mathrm{Mpc}^{-1}$ assuming integration time of $1000 \mathrm{~h}$ and $10 \mathrm{MHz}$ bandwidth (dashed black, bottom right).

temperature accounting for both the scattering term (as a function of the local value of $v_{\mathrm{b}-\mathrm{DM}}$ ) and the astrophysical heating rate. The $21-\mathrm{cm}$ signal is then calculated using the corresponding stellar Ly- $\alpha$ and ionizing backgrounds. We calculate the global signal (as observed by EDGES) averaging over the value of $v_{\mathrm{b}-\mathrm{DM}}$ which is drawn from the Maxwell-Boltzmann distribution. In addition, we estimate the rms of its fluctuations from the dark ages, cosmic dawn, and the epoch of reionization. For the purpose of this Letter we neglect the $21-\mathrm{cm}$ fluctuations due to the density and inhomogeneous astrophysical radiation fields, such as $\mathrm{x}$-ray and Ly- $\alpha$ backgrounds, due to the much larger fluctuations induced by the velocity-dependent cross section.

Results.-The magenta lines in the two bottom panels of Fig. 1 show the range at $z=17$ of the global signal $T_{21}(z)$ and the rms of the fluctuations expected from the entirety of the considered models, with and without the contribution from baryon-DM scattering. The variation on the sky is determined by the brightness temperature as a function of the local $v_{\mathrm{b}-\mathrm{DM}}$; this function is also shown in the figure (top panels). First, to highlight the importance of the scattering process we show an envelope of the maximal and minimal $T_{21}(z)$ as well as the maximal rms of the fluctuations for the 
entire ensemble of the 6389 astrophysical cases without baryon-DM scattering (these do include the astrophysical fluctuation sources), and 325839 cases including the scattering. The recent detection by EDGES low-band antenna[9] (the best-fit signal is shown in Fig. 1, yellow line) and the nondetection by EDGES high-band antenna [17] constrain the space of both astrophysical and DM parameters. Here we only verify a rough agreement with the data by imposing the following cuts based on the published $99 \%$ or $3 \sigma$ limits: The data collected by lowband, high-band antenna [17] rule out models with large variations and imply

$$
\left|T_{21}(100 \mathrm{MHz})-T_{21}(150 \mathrm{MHz})\right|<300 \mathrm{mK} .
$$

EDGES low-band data give a positive detection and require the absorption feature to be deep, broad, and localized within the band [9]. Within 99\% confidence, the cosmological signal should satisfy

$$
\begin{aligned}
300 \mathrm{mK}<\{ & \max \left[T_{21}(60<\nu<68)\right] \\
& \left.-\min \left[T_{21}(68<\nu<88)\right]\right\}<1000 \mathrm{mK},
\end{aligned}
$$

and

$$
\begin{aligned}
300 \mathrm{mK}< & \left\{\max \left[T_{21}(88<\nu<96)\right]\right. \\
& \left.-\min \left[T_{21}(68<\nu<88)\right]\right\}<1000 \mathrm{mK},
\end{aligned}
$$

There are 2448 models in total, all shown in Fig. 1 (gray curves), that satisfy both sets of conditions [Eqs. (1)-(3)]. The minimum and maximum points from Eqs. (2) and (3) are exemplified on the bottom left panel of Fig. 1. Another global signal experiment, LEDA [18], reported a $2 \sigma$ limit of $-890 \mathrm{mK}$ on the amplitude of $T_{21}$ at $z \sim 20$, which could also be used to rule out extreme cases of baryon overcooling. However, here we rely only on the EDGES data which provides stronger constraints.

We first examine all the considered models with and without baryon-DM scattering. Unlike in the cases of negligible scattering in which the shape of the signal is universal and is described by an absorption trough followed in some cases by an emission feature [6], the added parameter space of DM models contributes to a larger variety of shapes (e.g., multiple wiggles during the cosmic dawn absorption) for both the global signal and the rms of the fluctuation. The effect of DM on the absorption trough itself can be very strong, leading to an order of magnitude increase in the amplitude. Specifically, the deepest possible absorption in the case with no scattering is $T_{21}=-247 \mathrm{mK}$ at $120 \mathrm{MHz}$, while with the scattering the absorption trough can reach $T_{21}=-2180 \mathrm{mK}$ at $92 \mathrm{MHz}$. Additionally, the fluctuations are enhanced with the peak power shifts from $87.7 \mathrm{mK}$ at $153 \mathrm{MHz}$ to $855 \mathrm{mK}$ at $97 \mathrm{MHz}$. For the entire ensemble of models without baryon-DM scattering the most negative feature of the global signal during cosmic dawn and reionization $(6<z<35,39.5<\nu<202 \mathrm{MHz})$ is anywhere between $-247.15 \mathrm{mK}$ and $-8.02 \mathrm{mK}$ and is localized in the $9.1<z<35$ range $(39.5<\nu<140.6 \mathrm{MHz})$; while in all the considered scenarios with baryon-DM scattering the maximal absorption is between $-2179.2 \mathrm{mK}$ and $-2.1 \mathrm{mK}$ and can be located anywhere within the $6<z<35$ range. The strongest fluctuations are expected to have an rms amplitude between 1.5 and $87.7 \mathrm{mK}$ with the redshift of the peak power in the range $6.8<z<30$ $(45.8<\nu<182.1 \mathrm{MHz})$ in the models without scattering, and the maximal rms during cosmic dawn $(\nu<100 \mathrm{MHz}$, $z>13.2)$ is $25.3 \mathrm{mK}$. With scattering, the maximal fluctuation amplitude (due to baryon-DM scattering only) can be anywhere between 0 and $855.4 \mathrm{mK}$ at $6.3<z<35$. Finally, with the scattering affecting the energy budget, gas can be heated either faster or slower depending on the detailed balance between baryons and the dark sector. Specifically, we find that the redshift of the heating transition, i.e., the moment at which the brightness temperature transitions from absorption to emission during cosmic dawn or reionization, varies over a wider range when the scattering is included, and can be anywhere within $6<$ $z_{h}<35$ compared to $6<z_{h}<30.3$ in the case of no scattering.

Adding the EDGES constraints restricts both the amplitude and the position of the absorption trough. Namely, the absorption feature of the compatible models can only be as deep as $-1543 \mathrm{mK}$ to $-304 \mathrm{mK}$ and must peak in the narrow redshift range $15.6<z<20.1$ $(67.7<\nu<86.1 \mathrm{MHz})$. This deep absorption trough should be readily accessible to other global experiments such as SARAS-2 [19,20], LEDA [18,21], and SCI-HI/ PRIZM [22]. To agree with the observations, the range of $z_{h}$ is restricted to $8.7<z_{h}<17.7$. In other words, baryons cannot be significantly hotter than the CMB at $z \sim 17$ (the center of EDGES low-band region) and should heat up considerably at $z \sim 9-13$. In addition, extremely low heating efficiency is ruled out by both EDGES high-band antenna[23] and SARAS-2 [19,20]. Finally, the scenarios favored by EDGES yield fluctuations stronger by an order of magnitude than those previously predicted (assuming collisionless dark matter), with the peak rms between 8.07 and $807.3 \mathrm{mK}$ found at $14.6<z<21.5(63.5<\nu<$ $91.6 \mathrm{MHz}$ ). Such strong fluctuations are easily detectable by interferometric arrays such as HERA [24] and SKA [25], whose predicted thermal noise is $\sim 0.87 \mathrm{mK}$ at $z=17$ (Fig. 1, bottom left panel). A strong 21-cm signal is also possible during dark ages but it is expected to be at extremely low frequencies inaccessible from the ground [2].

Another statistic that can be measured from images taken by $21-\mathrm{cm}$ interferometers is the probability distribution function (PDF) of the $21-\mathrm{cm}$ brightness temperature (relative to the mean global temperature). If the function 
$T_{21}\left(v_{\mathrm{b}-\mathrm{DM}}\right)$ is monotonic, then this function can essentially be read off an observed PDF (assuming that the 21-cm fluctuations are indeed dominated by baryon-DM scattering), since the PDF of $v_{\text {b-DM }}$ itself is known to be Maxwellian [26]; however, the top right panel of Fig. 1 shows that in some cases this function is non-monotonic, so reconstructing it from the $21-\mathrm{cm}$ PDF will involve model fitting.

Baryonic acoustic oscillations (BAO).-The relative baryon-DM velocity is supersonic at recombination and, because of the coupling between baryons and photons prior to recombination, the velocity field bears a strong BAO signature [26]. In the absence of baryon-DM scattering, $v_{\mathrm{b}-\mathrm{DM}}$ can generate enhanced oscillations in the $21-\mathrm{cm}$ signal by modulating star formation in primordial halos $[27,28]$. With the baryon-DM scattering, as a result of the dependence of the cross section on $v_{\mathrm{b}-\mathrm{DM}}$, the $\mathrm{BAO}$ feature in the 21-cm signal is expected to be even stronger, acting as a smoking gun signature of baryon-DM scattering. The effect of $v_{\mathrm{b}-\mathrm{DM}}$ on the $21-\mathrm{cm}$ fluctuations during dark ages was first pointed out in Ref. [2], while Barkana [3] considered the scattering during cosmic dawn for the first time. An enhanced BAO pattern in the $21-\mathrm{cm}$ power spectrum detected by an interferometric array, such as HERA and SKA, would be a telltale signal of baryon-DM scattering and would verify the EDGES detection as well as its DM interpretation.

In Fig. 2 we demonstrate the BAO pattern seen in the power spectrum of the $21-\mathrm{cm}$ signal from $z=17$ (assuming that DM cooling dominates and other $21-\mathrm{cm}$ fluctuations can be neglected). To calculate the power spectrum we generated a distribution of the velocity field in units of the rms velocity (e.g., see Fig. 1 of Ref. [3]) in a comoving volume of $1.536^{3} \mathrm{Gpc}^{3}$. Next, for each model we

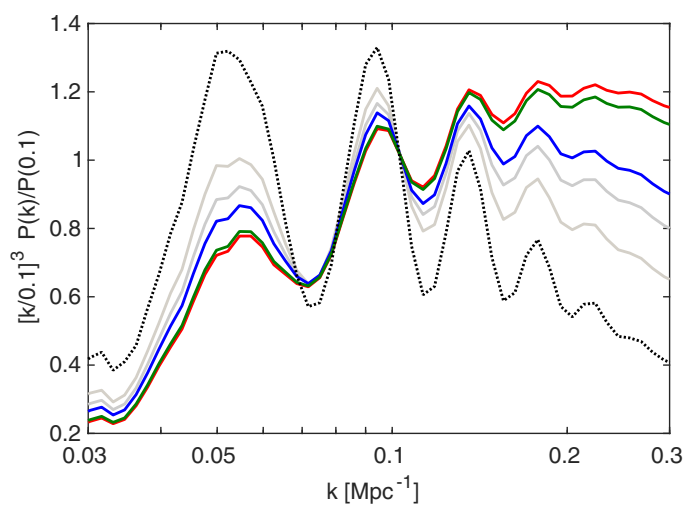

FIG. 2. Power spectrum of the 21-cm signal versus wave number at $z=17$. The spectra are shown for: the model with the strongest absorption (blue), the lowest $z_{h}$ (green) and the highest rms (red); in addition, several other random models from the ensemble compatible with EDGES are shown (gray). Also shown is the power spectrum of $v_{\mathrm{b}-\mathrm{DM}}$ (black dotted curve). In order to highlight the BAO shape, all the curves are normalized to unity at $k=0.1 \mathrm{Mpc}^{-1}$. transformed the velocity cube to the $21-\mathrm{cm}$ signal using the $v_{\mathrm{b}-\mathrm{DM}} \rightarrow T_{21}$ mapping from Fig. 1 (top row) and calculated the power spectrum. The resulting power spectra are shown in Fig. 2. To highlight the BAO shape (the rms amplitude was separately shown in Fig. 1) we show the power spectra relative to their value at $k=0.1 \mathrm{Mpc}^{-1}$ and average over 10 independent realizations of the initial velocity cubes to compensate for the cosmic variance effect on the largest scales.

Conclusions.-The recent detection by EDGES lowband antenna, if indeed cosmological, requires a new theoretical explanation beyond the standard astrophysical model. In this Letter, considering baryon-DM scattering as a viable mechanism to produce the observed absorption, we have surveyed the parameter space of astrophysical and DM properties. We have shown that the expected global signal and rms of the fluctuations vary over a much larger range than before. The velocity-dependent cross-section imprints enhanced $\mathrm{BAO}$ which could be used to constrain dark matter theories. In this work we assumed that all DM particles scatter off baryons. As laid out in Ref. [3], this is consistent with existing constraints that arise directly from the baryon-DM interaction itself. Therefore, the EDGES signal is unlikely to be the result of gas cooling via scattering with a dominant component of the DM. However, scenarios where a few percent of the DM particles are millicharged are allowed [29-31] and would generate similar features in the $21-\mathrm{cm}$ line as predicted here. Relative to our present results, the modification in these models would largely amount to an effective renormalization of $\sigma_{1}$. In addition, other DM scenarios may lead to baryon cooling: a small amount of condensed QCD axion dark matter could even explain EDGES results within the context of standard model [32,33].

For R. B. and A. C., this publication was made possible by the ISF-NSFC joint research program (Grant No. 2580/ 17) and through the support of a grant from the John Templeton Foundation; the opinions expressed in this publication are those of the authors and do not necessarily reflect the views of the John Templeton Foundation.

Note added.-Recently, scenarios in which all DM particles scatter off baryons through velocity-dependent interactions were ruled out, since this would require a light mediator that is highly constrained by 5th force experiments and limits from stellar cooling [29].

[1] H. Tashiro, K. Kadota, and J. Silk, Phys. Rev. D 90, 083522 (2014).

[2] J. B. Muñoz, E. D. Kovetz, and Y. Ali-Haïmoud, Phys. Rev. D 92, 083528 (2015).

[3] R. Barkana, Nature (London) 555, 71 (2018).

[4] S. A. Wouthuysen, Astron. J. 57, 31 (1952).

[5] G. B. Field, Proc. IRE 46, 240 (1958). 
[6] A. Cohen, A. Fialkov, R. Barkana, and M. Lotem, Mon. Not. R. Astron. Soc. 472, 1915 (2017).

[7] A. Cohen, A. Fialkov, and R. Barkana, arXiv:1709.02122.

[8] A. Cohen, A. Fialkov, R. Barkana, and R. A. Monsalve (to be published).

[9] J. D. Bowman, A. E. E. Rogers, R. A. Monsalve, T. J. Mozdzen, and N. Mahesh, Nature (London) 555, 67 (2018).

[10] C. Feng and G. Holder, Astrophys. J. 858, L17 (2018).

[11] D. J. Fixsen, A. Kogut, S. Levin, M. Limon, P. Lubin, P. Mirel, M. Seiffert, J. Singal, E. Wollack, T. Villela, and C. A. Wuensche, Astrophys. J. 734, 5 (2011).

[12] R. Subrahmanyan and R. Cowsik, Astrophys. J. 776, 42 (2013).

[13] T. Fragos, B. D. Lehmer, S. Naoz, A. Zezas, and A. Basu-Zych, Astrophys. J. Lett. 776, L31 (2013).

[14] A. Fialkov, R. Barkana, and E. Visbal, Nature (London) 506, 197 (2014).

[15] P. A. R. Ade et al. (Planck Collaboration), Adv. Appl. Phys. 594, A13 (2016).

[16] P. A. R. Ade et al. (Planck Collaboration), Adv. Appl. Phys, 571, A16 (2014).

[17] R. A. Monsalve, A. E. E. Rogers, J. D. Bowman, and T. J. Mozdzen, Astrophys. J. 847, 64 (2017).

[18] G. Bernardi, J. T. L. Zwart, D. Price, L. J. Greenhill, A. Mesinger, J. Dowell, T. Eftekhari, S. W. Ellingson, J. Kocz, and F. Schinzel, Mon. Not. R. Astron. Soc. 461, 2847 (2016).

[19] S. Singh, R. Subrahmanyan, N. Udaya Shankar, M. Sathyanarayana Rao, A. Fialkov, A. Cohen, R. Barkana, B. S. Girish, A. Raghunathan, R. Somashekar, and K. S. Srivani, Astrophys. J. Lett. 845, L12 (2017).

[20] S. Singh, R. Subrahmanyan, N. Udaya Shankar, M. Sathyanarayana Rao, A. Fialkov, A. Cohen, R. Barkana, B. S. Girish, A. Raghunathan, R. Somashekar, and K. S. Srivani, Astrophys. J. 858, 54 (2018).

[21] D. C. Price, L. J. Greenhill, A. Fialkov, G. Bernardi, H. Garsden, B. R. Barsdell, J. Kocz, M. M. Anderson,
S. A. Bourke, J. Craig, M. R. Dexter, J. Dowell, M. W. Eastwood, T. Eftekhari, S. W. Ellingson, G. Hallinan, J. M. Hartman, R. Kimberk, T. J. W. Lazio, S. Leiker et al., arXiv: 1709.09313.

[22] T. C. Voytek, A. Natarajan, J. M. Jáuregui García, J. B. Peterson, and O. López-Cruz, Astrophys. J. Lett. 782, L9 (2014).

[23] R. A. Monsalve, A. Fialkov, J. D. Bowman, A. E. E. Rogers, T. J. Mozdzen, A. Cohen, R. Barkana, and N. Mahesh (to be published).

[24] D. R. DeBoer, A. R. Parsons, J. E. Aguirre, P. Alexander, Z. S. Ali, A. P. Beardsley, G. Bernardi, J. D. Bowman, R. F. Bradley, C. L. Carilli, C. Cheng, E. de Lera Acedo, J. S. Dillon, A. Ewall-Wice, G. Fadana, N. Fagnoni, R. Fritz, S. R. Furlanetto, B. Glendenning, B. Greig et al., Publ. Astron. Soc. Pac. 129, 045001 (2017).

[25] L. Koopmans, J. Pritchard, G. Mellema, J. Aguirre, K. Ahn, R. Barkana, I. van Bemmel, G. Bernardi, A. Bonaldi, F. Briggs, A. G. de Bruyn, T. C. Chang, E. Chapman, X. Chen, B. Ciardi, P. Dayal, A. Ferrara, A. Fialkov, F. Fiore, K. Ichiki et al., Proc. Sci., AASKA14 (2015) 001.

[26] D. Tseliakhovich and C. Hirata, Phys. Rev. D 82, 083520 (2010).

[27] N. Dalal, U.-L. Pen, and U. Seljak, J. Cosmol. Astropart. Phys. 11 (2010) 007.

[28] E. Visbal, R. Barkana, A. Fialkov, D. Tseliakhovich, and C. M. Hirata, Nature (London) 487, 70 (2012).

[29] R. Barkana, N. J. Outmezguine, D. Redigolo, and T. Volansky, arXiv:1803.03091.

[30] A. Berlin, D. Hooper, G. Krnjaic, and S. D. McDermott, following Letter, Phys. Rev. Lett. 121, 011102 (2018).

[31] J. B. Muñoz and A. Loeb, arXiv:1802.10094.

[32] P. Sikivie, arXiv:1805.05577.

[33] N. Houston, C. Li, T. Li, Q. Yang, and X. Zhang, arXiv: 1805.04426.

[34] G. D'Amico, P. Panci, A. Strumia, this issue, Phys. Rev. Lett. 121, 011103 (2018). 